\title{
DatAR: Your brain, your data, on your desk - A research proposal
}

\author{
Ghazaleh Tanhaei \\ Information and Computing Sciences \\ Utrecht University \\ Utrecht, The Netherlands \\ g.tanhaei@uu.nl
}

\author{
Lynda Hardman \\ Centrum Wiskunde \& Informatica \\ Utrecht University \\ Amsterdam/Utrecht, The Netherlands \\ Lynda.Hardman@cwi.nl
}

\author{
Wolfgang Huerst \\ Information and Computing Sciences \\ Utrecht University \\ Utrecht, The Netherlands \\ huerst@uu.nl
}

\begin{abstract}
We present a research proposal that investigates the use of 3D representations in Augmented Reality (AR) to allow neuroscientists to explore literature they wish to understand for their own scientific purposes. Neuroscientists need to identify potential real-life experiments they wish to perform that provide the most information for their field with the minimum use of limited resources. This requires understanding both the alreadyknown relationships among concepts and those that have not yet been discovered. Our assumption is that by providing overviews of the correlations among concepts through the use of linked data, these will allow neuroscientists to better understand the gaps in their own literature and more quickly identify the most suitable experiments to carry out. We will identify candidate visualizations and improve upon these for a specific information need. We describe our planned prototype 3D AR implementation and directions we intend to explore.
\end{abstract}

Index Terms-Immersive analytics, augmented reality, linked data, neuroscience publication exploration

\section{INTRODUCTION}

Neuroscientists work in a highly complex field where they need to explore results from many literature sources for different tasks. An example task is to identify potential future experiments to carry out. For this, they need to understand the relationships between high-level neuroscientific concepts, such as brain diseases, brain regions and cognitive functions. To further understanding in the field, neuroscientists perform experiments to understand relationships between concepts such as a specific brain region and related brain diseases. Confirming these relationships using empirical studies has an enormous cost in terms of both time and money, making reliable identification of high-potential experiments a priority.

The Brain-inspired Intelligence research group ${ }^{1}$ uses a linked data repository ${ }^{2}$ to represent the results of extracting

1 http://bii.ia.ac.cn, Chinese Academy of Sciences, Institute of Automation, Beijing, http://english.ia.cas.cn/ and connecting concepts from neuroscience publications ${ }^{3}$. This contains titles and abstracts of published medical papers gathered from many sources, such as PubMed ${ }^{4}$. To decide on an experiment to carry out, a neuroscientist needs to understand existing relationships reported in the literature, and whether there is a lack of experimental evidence rather than a lack of causality.

While neuroscientists have access to all the information in the repository, currently, they require technical skills for exploring the information for their research task. For example, they currently require the ability to write $\mathrm{SPARQL} \mathrm{L}^{5}$ queries to access the information contained in the linked data repository. Our aim is to provide an interactive environment that allows neuroscientists to explore the repository in a way that more directly supports their literature exploration task.

Augmented reality (AR) enables a form of visualization and exploration that can potentially reduce the barriers between users and their data allowing them to gain insights based on the data more easily $[1,2,18,19]$. To empower neuroscientists working in their own field, we propose the use of AR to display relationships among relevant literature represented in the Linked Brain Data (LBD) repository in our prototype environment DatAR: Data visualization by means of Augmented Reality. Our motivation is to allow neuroscientists to formulate high-level queries, with the assistance of clustering techniques and 3D displays of the patterns of the relationships found.

This paper describes the context of our research, in particular, the literature exploration task of neuroscientists and

\footnotetext{
${ }^{2}$ For an introduction, see [24]

${ }^{3} \mathrm{http}: / / w w w . l i n k e d-b r a i n-d a t a . o r g$

${ }^{4}$ https://www.ncbi.nlm.nih.gov/pubmed/

${ }^{5} \mathrm{SPARQL}$ is a W3C language for querying linked data $[21,22]$
} 
illustrates our ideas on how to support this. Our envisaged contributions can be summarized as follows:

- Providing an effective 3D visualization of concepts in the neuroscience literature, such as brain regions and brain diseases, and their complex relationships to allow neuroscientists to better understand them.

- Presenting 3D visualizations in AR with interactive support for neuroscientists to explore these relations for their task of identifying a suitable experiment.

Note that while the DatAR system will be able to identify correlations between concepts, this does not guarantee the existence of a causal relation - it is the expert neuroscience user who forms an understanding through exploration of the AR representations.

\section{Related Work}

A majority of studies on exploration and visualization in the domain of immersive analytics (IA) focus on numeric data or using 3D platforms other than AR. We claim that DatAR is novel in the area of IA by combining linked data, clustering and AR. We present related work in visualization platforms for numeric and for linked data since we use a combination of these.

\section{A. Visualization Platforms for Numeric Data in VR/AR}

ImAxes is an immersive system that visualizes multivariate numeric data in VR, Fig. 1 [3]. Users can manipulate and combine axes through modeless, fluid interactions. Working with axes allows novice users to create new forms of plots with little cognitive overhead. Direct manipulation of objects within the virtual environment minimizes the effort for users to start working with the system. IATK is a toolkits the creation of a range of immersive visualizations, Fig. 2 [4].

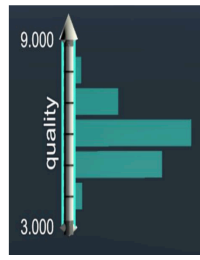

(a) Histogram

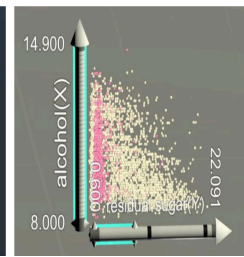

(b) 2D Scatterplot

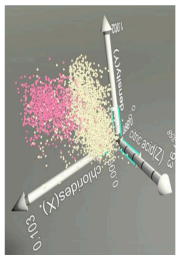

(c) 3D Scatterplot

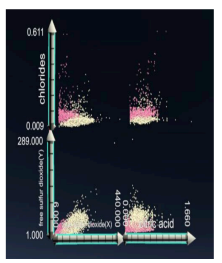

(d) SPLOM

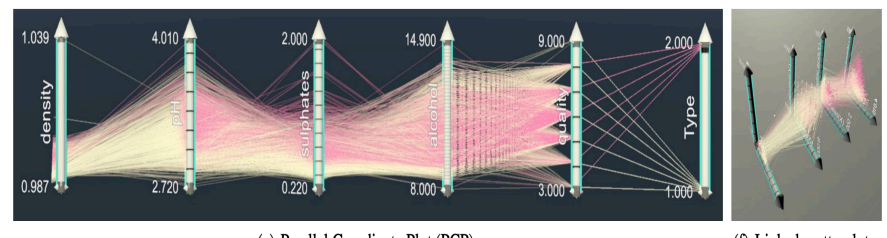

(e) Parallel Coordinate Plot (PCP)

(f) Linked scatterplots

Fig. 1. 2D and 3D visualizations in ImAxes [3] (reproduced with permission).

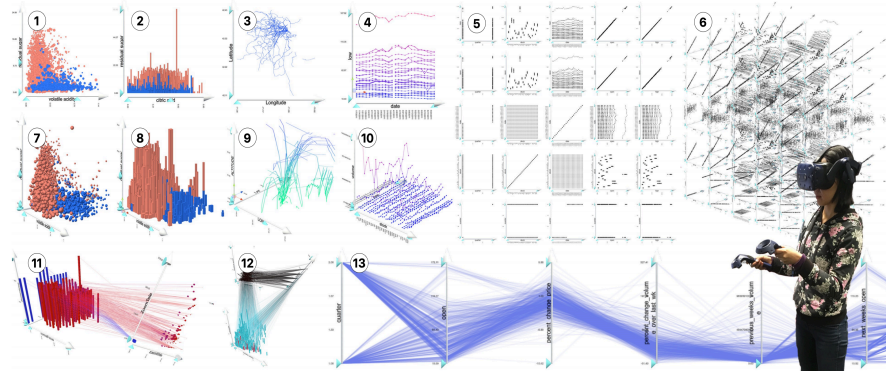

Fig. 2. Multidimensional visualizations in IATK [4] (reproduced with permission).

Users can use IATK to create many forms of visualizations in a virtual environment, such as scatterplots and parallel coordinate plots, in addition to visualizations of continuous, discrete and linked data. Since there are some commonalities with our work in terms of visualization type and display environment, the technical features and design requirements are useful for our tasks. The results that we are going to present have 3D positions and a scatterplot can illustrate these results as nodes. Also, we need an environment (e.g. real) as a display that can show each of nodes positions clearly in order to help user to freely observe different dimensions of plot and detect close vicinity nodes.

The DXR project provides a toolkit for a wide range of users (from advanced programmers to non-programmers) [5]. The toolkit was introduced for developers with no experience in 3D graphs, AR and VR to create graphs easily and quickly in a GUI. A grammar is used to specify a visual mapping declaratively, with live updates in the visualization [23]. This type of grammar can be used to load our 3D position data and define its properties like shape of nodes.

\section{B. Visualization Platforms for Linked Data in $2 D \& 3 D$}

Tarsier [7] supports teachers with introducing formalisms of semantic web data representations to students. Similar to our work, it represents linked data in a 3D environment. Tarsier is based on the idea of spatially distancing data points into different semantic planes. For example, a user could decide to place brain diseases, brain regions and cognitive functions each on their own plane. The 3D visualization shows links between semantic planes, so users can easily find different views to explore the data.

In SuperSQL [16], the user submits a query by describing the data they want to retrieve and the layout of the data representation. After parsing a SuperSQL query to an SQL query, a generated XML file is passed to Unity. This allows users to export 3D models of their query results. The limitations are the lack of interactivity (an object cannot be manipulated by the user) and the fixed position of objects (the position of objects is influenced only by the hierarchy of the data). Passing a query to Unity is a useful technique, where we interpret the result of a SPARQL query based on the user's interaction in the $3 \mathrm{D}$ environment and display the resulting data file in Unity. 
A visualization of linked data should be easily readable and understandable [10] and help users understand the information expressed within the (highly) connected graphs [8]. The user interface should support users in exploring the graphs [9]. Our goal is to provide an interactive $3 \mathrm{D}$ AR environment that supports a neuroscientist in exploring relationships, represented within the linked brain data repository, among concepts and their correlations in relevant literature.

\section{DATAR UI DESIGN ReQUiREMENTS}

The design requirements we identify below are based on our users' needs and the presentation of data graphs. Since these graphs can be very complex, our proposed visualization tool needs to satisfy the requirements listed below.

\section{A. Node shape}

Since we have about 300 nodes with the names of concepts in the graph, we should consider an appropriate shape for them. The shape of nodes ${ }^{6}$ needs to allow users access to the name of the concept ${ }^{7}$ and also perceive the position of the named concept in relation to large numbers of nodes when presenting the results of queries.

\section{B. Node position}

Node position can be used to represent semantic similarity between concepts. For instance, morphine dependence and drug dependence are closely related semantically so they should be presented close to each other.

\section{Node size}

The number of co-occurrences of a term indicates the number of times that a concept, such as a specific brain disease, appears in the medical literature together with another concept, such as a brain region. For example, the number of co-occurrences of Amygdala and Alzheimer in the LBD graph is 238 . The size of a node in the graph can be used to indicate the number of co-occurrences, where this could, for example, be proportional to the number of co-occurrences or indeed inversely proportional to indicate the importance of unexplored areas.

\section{Result filtering}

As the number of query results can be high, users should be able to filter them based on the number of co-occurrences. For neuroscientists who are seeking a potential research topic, it is important to be able to find relations with few or even zero co-occurrences as well as those with large ones.

\section{METHOD}

User Scenario-Diseases with just a small number of, or even zero, co-occurrences with a specific part of the brain in the literature can be interesting, since they may indicate unexplored areas that need further research. Investigating

\footnotetext{
${ }^{6}$ Nodes in the graph represent concepts from the neuroscience literature and are used for presenting the results of a query.

${ }^{7}$ One of the concepts is brain disease.
}

diseases with a high number of co-occurrences may also be important since researchers need to verify others' findings. In this scenario we present small numbers of co-occurrences to help users uncover unexplored relations. To describe the problem of finding new concepts, we consider a scenario where a user is interested in diseases related to the Amygdala. We select the Amygdala as an example, since it is a part of the brain that has relationships with about 107 diseases, which is neither very many nor very few.

Our assumption is that brain diseases that often co-occur in the same publications relate to the same regions of the brain. We define these diseases as being semantically similar and assume that they affect similar regions of the brain. To identify groups of semantically similar diseases we propose to use the Latent Dirichlet Allocation (LDA) method, which assumes that all of the words in one document are likely to be related to the same topic $[12,13,14]$. We expect neighboring brain diseases can affect one specific brain regions to be semantically similar. Using LDA we can assign an $\mathrm{n}$ dimensional position for each brain disease in the document collection.

Humans, however, cannot (easily) perceive more than three spatial dimensions, so for visualization purposes, we need to reduce the number of dimensions to three. We should preserve the structure of low-dimensional data such as highdimensional by t-distributed Stochastic Neighbor Embedding (t-SNE) and visualize them in order to find neighboring diseases [11]. Our colleagues in the Brain-inspired Intelligence group applied the LDA and t-SNE algorithms to about 300 diseases to find their 3D locations, Fig. 4. 


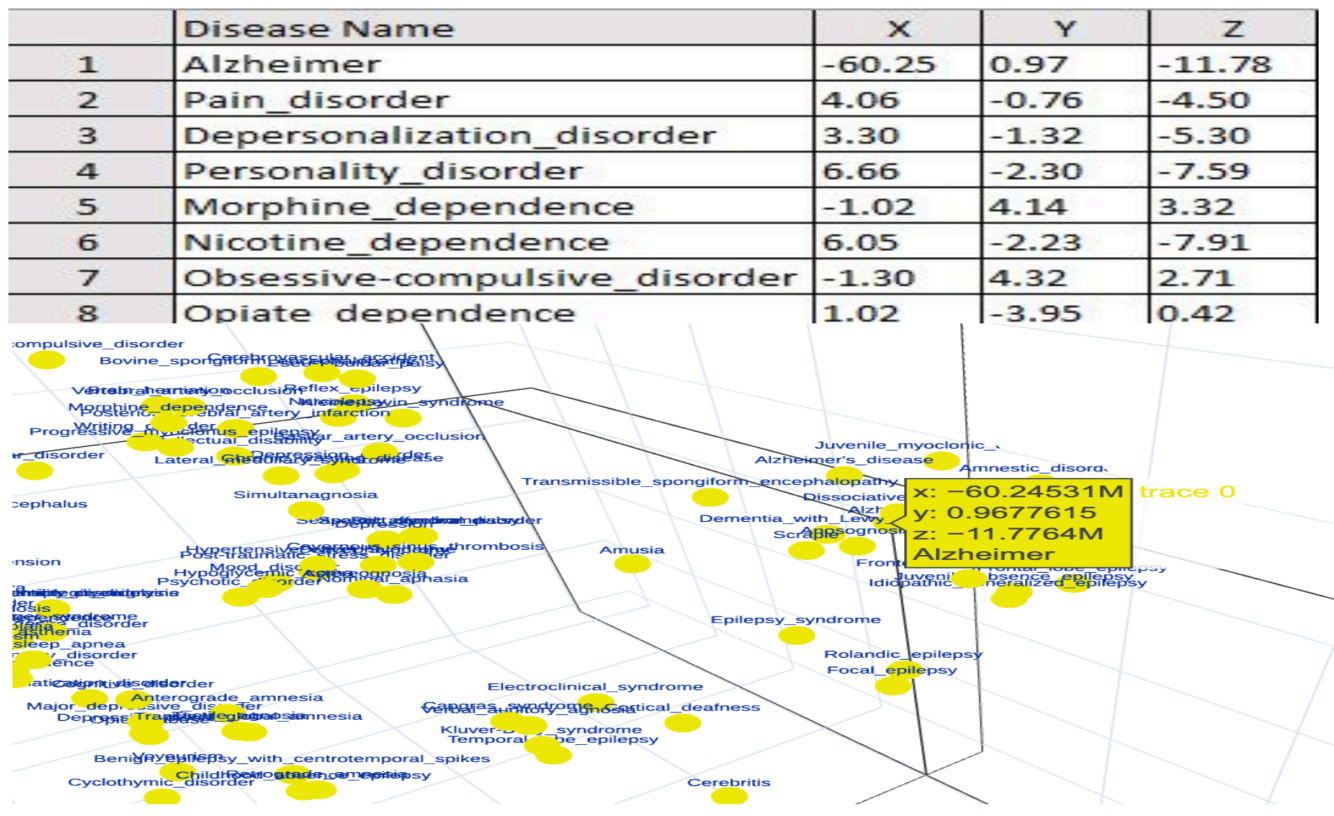

Fig.4. 3D position of brain diseases generated by LDA and t-SNE algorithms. a. 3D coordinates of diseases. b. 3D location of diseases in graph by Plotly [17].

To help satisfy the goals of perception and memorability, we use a $3 \mathrm{D}$ brain disease graph and a 3D model of the brain that will be presented in an AR environment.

In our scenario, a user selects the Amygdala brain region and it will be highlighted in the 3D brain model. The user then filters the results on the basis of the number of co-occurrences. These are indicated in the 3D graph representation and can be filtered, for example, using a slider with two pointers (in this scenario the range can be from 1 to 1,303). The user can select a range, for example by using a drag gesture, for example, reducing the range of co-occurrences to between 3 and 10 . The resulting visualization shows 35 brain diseases that cooccur 3 to 10 times with the Amygdala in the 3D disease graph. Diseases that have a relation with Amygdala in the chosen range (3 to 10) will be presented with a distinctive visualization, for example node color.

In our scenario, our user wants to find infrequent relations in the data set. The 3D locations of diseases support the user in this aim. When the user selects a region in the 3D brain and desired range of co-occurrences, they can see the disease highlighted in the 3D disease graph. For a user it is important to observe the positions of diseases and their neighbors in the $3 \mathrm{D}$ graph precisely. Presenting $3 \mathrm{D}$ graphs in an AR environment enables users to find similar locations of related diseases by interacting with the graph (e.g., zooming in or rotating it) and see centralized nodes near the results. Finding diseases that are not part of the search results, but which are close to those related to that part of the brain would be a promising area for investigation. Fig.5 illustrates a mock-up version of this user scenario.

\section{DATAR ARCHITECTURE}

In the frontend, an AR platform enables the visualization of the results. In the backend, the system supports SPARQL querying of the LBD repository and machine learning methods. Both the frontend visualization and backend processing are intended to empower the neuroscientists' exploration of the publication collection. The DatAR frontend will be implemented in Unity, a platform for developing AR projects among others [6]. Fig. 6 presents an overview of the system architecture. 


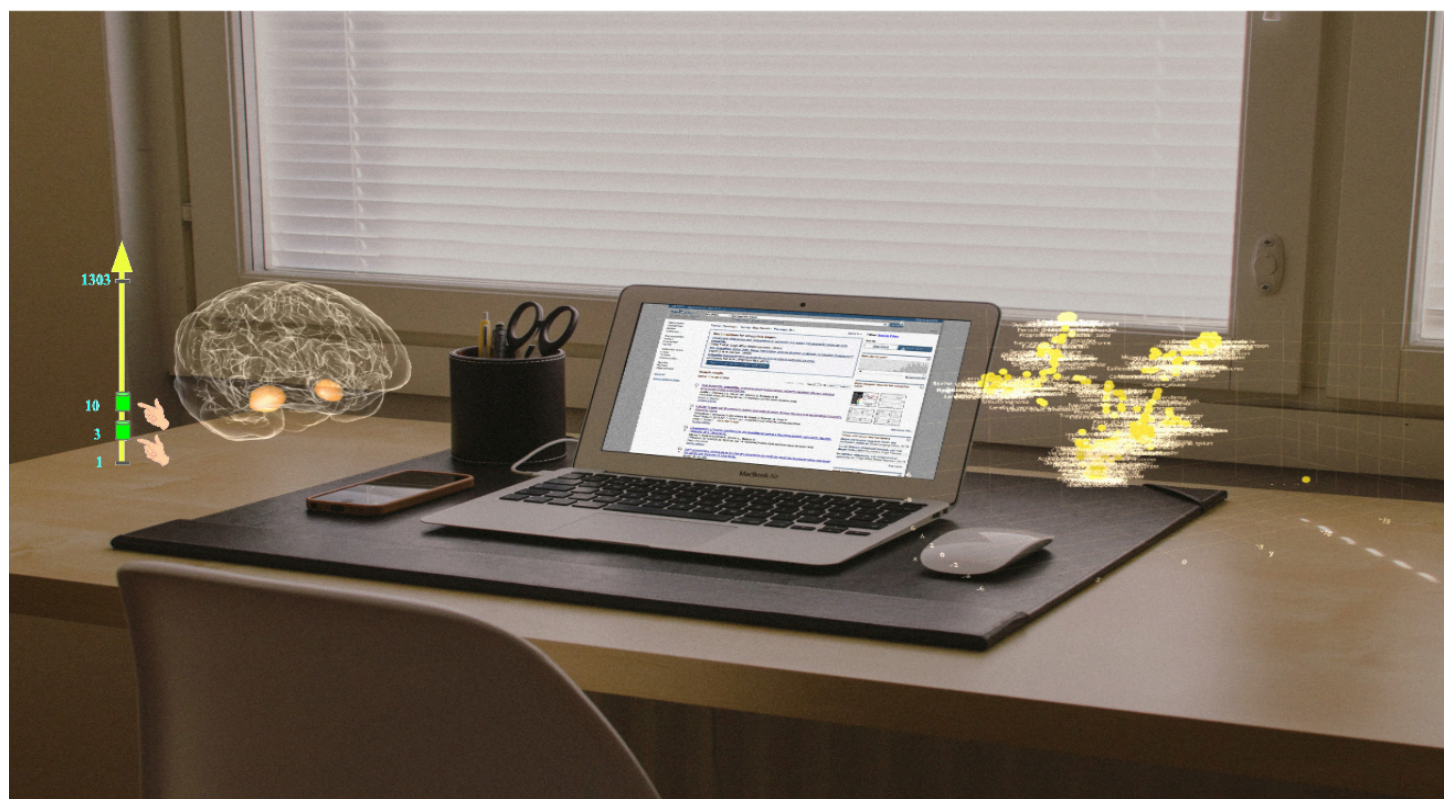

Fig. 5. A mock-up of DatAR. The left-hand side shows a 3D model of the brain [20] with the region selected by the user, Amygdala, highlighted and a slider for selecting the specific range of the number of co-occurrences. The right-hand side displays all the brain diseases in a 3D graph, although does not highlight which are specifically related to the selected region.

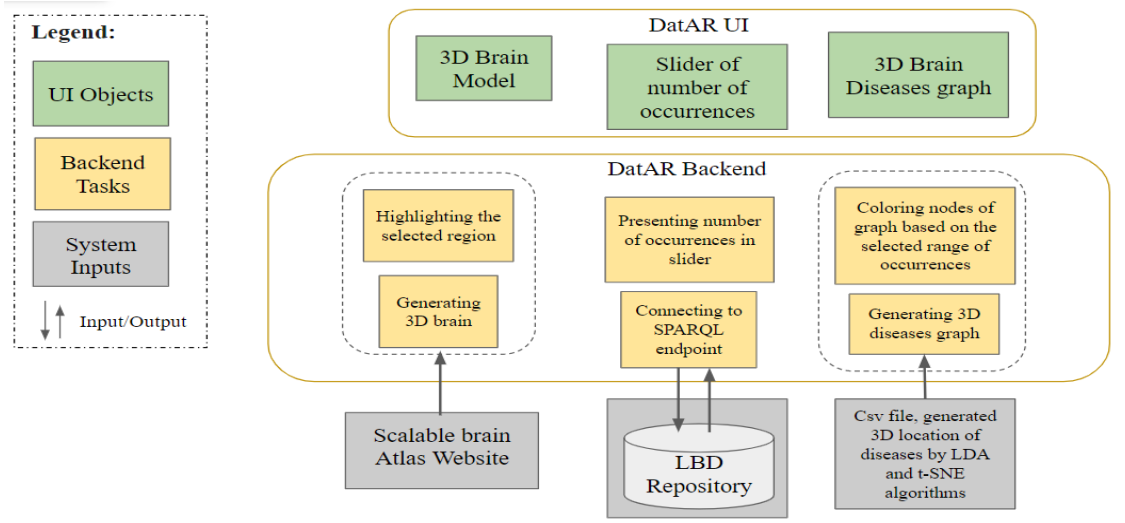

Fig. 6. System Overview.

Based on our research questions we will first carry out iterative rounds of scenario and user interface development before evaluating our work using usability and qualitative factors. We will specify a list of user tasks and check the number of tasks that users are able to complete successfully. The tasks will be chosen based on some criteria. As an example, an important feature of AR systems is the ability of frame-to-frame hand positioning and gesture tracking [15]. To ensure that the concentration flow of neuroscientists is not disrupted by the interaction within the system we will investigate the appropriateness of specific gestures.

\section{REASONS FOR USING AR}

Since this project is based on a 3D representation of diseases and a neuroscientist is going to make the final conclusions based on finding a neighboring disease, a clear representation of this graph is crucial. For example, moving

the mouse or zooming in or out can lead to different conclusions for a specific disease in the graph, Fig. 7. Allowing the user to manipulate the 3D disease graph in AR, for example by controlling the viewing angle, zooming in/out and rotating, will enable the user to easily identify neighboring diseases.

\section{NEXT STEPS}

Our hypothesis is that spatial proximity of brain diseases in relation to a specific brain region will help expert neuroscientists understand their research field better. Furthermore, AR displays have the potential to facilitate neuroscientists to easily explore and obtain insights from the data more effectively than by utilizing $2 \mathrm{D}$ visualizations or $3 \mathrm{D}$ visualizations projected onto a $2 \mathrm{D}$ display. Our next step is to implement the Amygdala user scenario in Unity 3D employing a head mounted display and controller to explore the effectiveness of the interface for neuroscientists. 

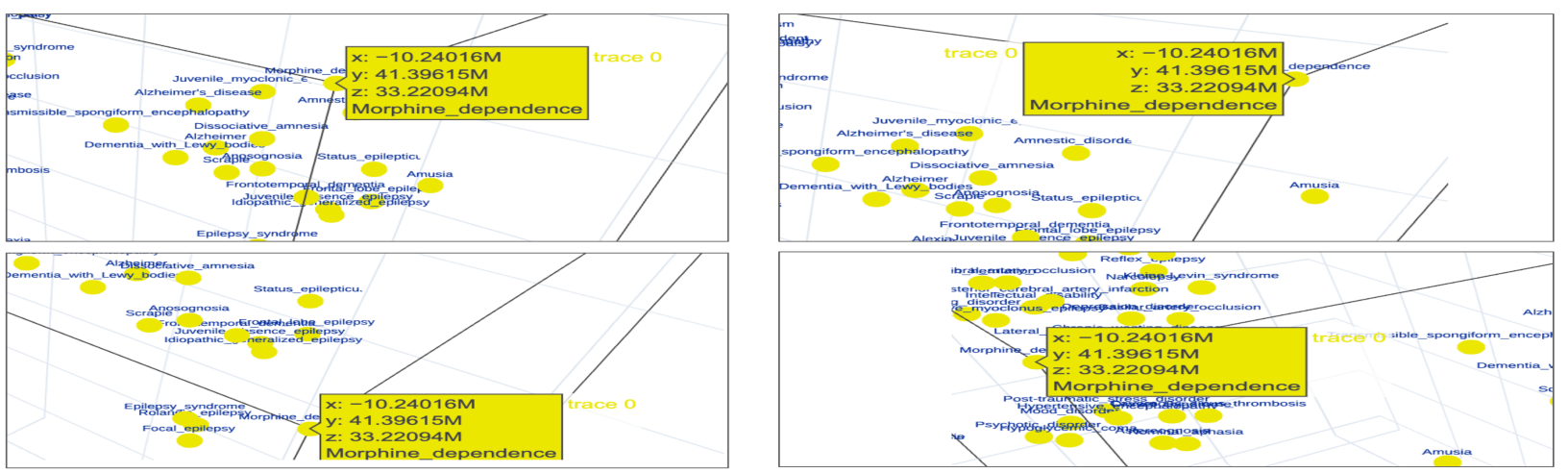

Fig. 7. Four 2D representations of a 3D diseases graph. Different neighbors for Morphine Dependence disease [17].

\section{ACKNOWLEDGMENTS}

We thank the research center for Brain-inspired Intelligence for making the Linked Brain Data available and, in particular, Cunqing Huangfu for sharing his insights as a neuroscientist. We also thank him for his technical help and, together with Ivar Troost, Utrecht University, for their valuable comments.

This research was supported by the Ministry of Science, Research and Technology of Iran.

\section{REFERENCES}

[1] K.Marriott, F.Schreiber, T.Dwyer, K.Klein, N.H. Riche, T.Itoh, W.Stuerzlinger, and B. H. Thomas, "Immersive Analytics," Springer, 1st ed. 2018.

[2] T. Chandler, M. Cordeil, T. Czauderna, T. Dwyer, J. Glowacki, C. Goncu, M. Klapperstueck, K. Klein, K. Marriott, F. Schreiber, and E. Wilson, "Immersive Analytics," Big Data Visual Analytics (BDVA), 2015, pp. 1-8.

[3] M. Cordeil, A. Cunningham, T. Dwyer, B. H. Thomas, and K. Marriott, "ImAxes: Immersive Axes as Embodied Affordances for Interactive Multivariate Data Visualisation," $30^{\text {th }}$ annual symposium on User Interface Software and Technology symposium, At Quebec City, Canada, pp. 71-83, October 2017.

[4] M. Cordeil, A. Cunningham, B. Bach, C. Hurter, B. H. Thomas, K. Marriott, and T. Dwyer, "IATK: An Immersive Analytics Toolkit," IEEE Conference on Virtual Reality and 3D User Interfaces (VR), March 2019.

[5] R. Sicat, J. Li, J.Y. Choi, M. Cordeil, W. Jeong, B. Bach, and H. Pfister, "DXR: A Toolkit for Building Immersive Data Visualizations," IEEE Transactions on Visualization and Computer Graphics, vol. 25, pp. 715 725, January 2019.

[6] https://unity.com.

[7] F. Viola, L. Roffia, F. Antoniazzi, A. D’Elia, C. Aguzzi, T. Cinotti, "Interactive 3D Exploration of RDF Graphs through Semantic Planes. Future Internet," Future Internet, Vol 10, August 2018.

[8] R. Chawuthai, and H. Takeda, "RDF Graph Visualization by Interpreting Linked Data as Knowledge," Computing the Semantic Similarity of Resources in DBpedia for Recommendation Purposes, pp.23-39, 2016.

[9] L. Zemmouchi-Ghomari, and A. Réda Ghomari, "Translating Natural Language Competency Questions into SPARQLQueries: A Case
Study," The First International Conference on Building and Exploring Web Based Environments, pp. 81-86, 2013.

[10] B. Schwendimann, "Concept maps as versatile tools to integrate complex ideas: From Kindergarten to higher and professional education", Journal of E-Learning and Knowledge Society, Vol 7, March 2015.

[11] L. van der Maaten and G. Hinton, "Visualizing Data using t-SNE", Journal of Machine Learning Research, Vol 9, 2008.

[12] S. Arora, R. Ge, Y. Halpern, D. M. Mimno, A. Moitra, D. Sontag, Y. $\mathrm{Wu}$, and M. Zhu, "Learning topic models-provably and efficiently," Commun ACM, pp. 85-93, 2018.

[13] G. Qi, J. Wu,Y. Zhou,Y. Du,Y. Jia, N. Hounsell, and N. A. Stanton, "Recognizing driving styles based on topic models", Elsevier Journal, Vol 66, pp. 13-22, January 2019.

[14] H. Jelodar, Y. Wang, C. Yuan, X. Feng, X. Jiang, Y. Li, and L. Zhao, "Latent Dirichlet allocation (LDA) and topic modeling: models, applications, a survey," Journal of Multimedia Tools and Applications, Vol 78, pp. 15169-15211, 2019.

[15] T. B. Moeslund, and N. Lau, "A brief overview of hand gestures used in wearable human computer interfaces," Tech. Rep Computer Vision and Media Technology Lab, Aalborg University, DK, 2003.

[16] T. Fujimoto, K. Goto, and M. Toyama, "3D Visualization of data using SuperSQL and Unity", Proceedings of the 22nd International Database Engineering \& Applications Symposium, pp. 141-147, 2018.

[17] https://plot.ly.

[18] P.A.Rauschnabel, A.Rossmann, M.C.Tom Dieck, “An adoption framework for mobile augmented reality games: The case of Pokémon Go," Computers in Human Behavior, Vol.76, pp.276-286, November 2017.

[19] S.A. Hirve, A. Kunjir, B. Shaikh, "An approach towards data visualization based on AR principles," International Conference on Big Data Analytics and Computational Intelligence (ICBDAC), 2017.

[20] https://scalablebrainatlas.incf.org.

[21] J. Pérez, M. Arenas, C. Gutierrez, "Semantics and complexity of SPARQL," J. ACM Transactions on Database Systems, Vol. 34, August 2009.

[22] B. Quilitz, U. Leser, "Querying Distributed RDF Data Sources with SPARQL," European Semantic Web Conference, Vol 5021, pp. 524$538,2008$.

[23] A. Satyanarayan, D. Moritz, K. Wongsuphasawat and J. Heer, "VegaLite: A Grammar of Interactive Graphics," IEEE Transactions on Visualization and Computer Graphics, vol. 23, pp. 341-350, January 2017.

[24] G. Antoniou, P. Groth, F. van Harmelen and R. Hoekstra, "A Semantic Web primer", Cambridge, Mass: MIT Press, 3rd ed, 2012. 